\title{
The Quaternionic Commutator Bracket and Its Implications
}

\author{
Arbab I. Arbab ${ }^{1,+}\left(\mathbb{D}\right.$ and Mudhahir Al Ajmi ${ }^{2, *}$ (i) \\ 1 Department of Physics, Faculty of Science, University of Khartoum, P.O. Box 321, Khartoum 11115, Sudan; \\ aiarbab@uofk.edu \\ 2 Department of Physics, College of Science, Sultan Qaboos University, P.O. Box 36, P.C. 123, \\ Muscat 999046, Sultanate of Oman \\ * Correspondence: mudhahir@squ.edu.om \\ + Current address: Department of Physics, College of Science, Qassim University, Qassim 51452, Saudi Arabia.
}

Received: 11 August 2018; Accepted: 9 October 2018; Published: 16 October 2018

check for updates

\begin{abstract}
A quaternionic commutator bracket for position and momentum shows that the quaternionic wave function, viz. $\widetilde{\psi}=\left(\frac{i}{c} \psi_{0}, \vec{\psi}\right)$, represents a state of a particle with orbital angular momentum, $L=3 \hbar$, resulting from the internal structure of the particle. This angular momentum can be attributed to spin of the particle. The vector $\vec{\psi}$, points in an opposite direction of $\vec{L}$. When a charged particle is placed in an electromagnetic field, the interaction energy reveals that the magnetic moments interact with the electric and magnetic fields giving rise to terms similar to Aharonov-Bohm and Aharonov-Casher effects.
\end{abstract}

Keywords: commutator bracket; quaternions; magnetic moments; angular momentum; quantum mechanics

\section{Introduction}

In quantum mechanics, particles are described by relativistic or non-relativistic wave equations. Each equation associates a spin state of the particle to its wave equation. For instance, the Schrödinger equation applies to the spinless particles in the non-relativistic domain, while the appropriate relativistic equation for spin-0 particles is the Klein-Gordon equation. The relativistic spin- $1 / 2$ particles are governed by the Dirac equation. Recall that this equation reduces, in the non-relativistic limit, to the Schrödinger-Pauli equation [1,2]. Particles with spin-1 are described by Maxwell equations. Charged particles interact with the electromagnetic field. The effect of the interaction of these particles is obtained via the minimal coupling ansatz, where the momentum of the free particle $\left(p_{\mu}\right)$ is replaced by $p_{\mu}+q A_{\mu}$, where $A_{\mu}$ is the photon field.

With this prescription, the Dirac equation as well as the Schrödinger-Pauli equation predict the existence of spin for the electron through the interaction of its magnetic moment with a magnetic field present. Thus, the spin angular momentum is deemed to be a quantum phenomenon having no classical analogue. Hence, the spin is an intrinsic property of a quantum particle. Therefore, the spin is not a result of the rotation of a point quantum particle (like the electron). It could be associated with the space in which the particle is defined. In our new formulation, the particle is defined by the scalar and vector wave functions. The spin angular momentum can be defined in terms of these vector and scalar wave functions.

In a recent paper, we have unified the above three quantum wave equations in a single equation. We call this equation a unified quantum wave equation [3]. In the present work, we would like to investigate the nature of spin relying on our unified quantum wave equation, and, using the quaternionic commutator bracket between position and momentum operators, viz., $\left[x_{i}, p_{j}\right]=i \hbar \delta_{i j}[2]$. This bracket is a fundamental cornerstone in formulating quantum mechanics. We would like here to 
derive some physics from this commutator algebra. It is argued that some aspects of standard model particle physics can be derived from an algebra and that some physical concepts such as particles, causality, and irreversible time may result from the algebra acting on itself [4].

Such a generalization led to interesting physical results pertaining to the nature of the wave equation describing quantum particles. We have found that an intrinsic angular momentum, and related to its internal nature a quantum particle, is associated with the quaternionic wave function $\widetilde{\psi}$ that we recently found. It is generally understood that spin is not due to the point-particle rotation. This is true if we treat a particle as a point particle. However, we have recently shown that the wavy nature of a quantum particle due to its wavepacket nature undergoes an internal rotation [3]. A wavepacket consists of two waves moving in opposite directions with speed of light. If a quantum particle is thought of a wavepacket or an extended object, then an internal rotation is plausible.

Our current investigations revealed that the scalar $\left(\psi_{0}\right)$ and vector $(\vec{\psi})$ wave functions describe a particle with internal orbital angular momentum, $L=3 \hbar$; and that the vector $\vec{\psi}$ is directed a long the direction of $\vec{L}$. This internal angular momentum can be attributed to some kind of spin. These may define the helicity states of the quantum particle. Such states can be compared with a spinor representation of the Dirac equation. When a charged particle is placed in an electromagnetic field, the magnetic moments interact with both electric and magnetic fields giving rise to Aharonov-Bohm and Aharonov-Casher effects. Recall that the angular momentum is normally associated with an external motion of a particle relative to some position. However, in our present case, the angular momentum is due to an internal reference frame. It is analogous to isospin, which was introduced for nuclear particles where the particle is assumed to be rotating in an abstract space.

\section{The Fundamental Commutator Bracket}

We have shown recently that a quaternionic eigne-value equation $\tilde{P} \tilde{\Psi}=m c \tilde{\Psi}$, where $\widetilde{P}=$ $\left(i \frac{E}{c}, \vec{p}\right), \widetilde{\psi}=\left(\frac{i}{c} \psi_{0}, \vec{\psi}\right)$, yields the unified quantum wave equations being [5]

$$
\begin{gathered}
\vec{\nabla} \cdot \vec{\psi}-\frac{1}{c^{2}} \frac{\partial \psi_{0}}{\partial t}-\frac{m_{0}}{\hbar} \psi_{0}=0, \\
\vec{\nabla} \psi_{0}-\frac{\partial \vec{\psi}}{\partial t}-\frac{m_{0} c^{2}}{\hbar} \vec{\psi}=0,
\end{gathered}
$$

and

$$
\vec{\nabla} \times \vec{\psi}=0 .
$$

In the ordinary quantum mechanics, a particle is described by a scalar or spinor; however, a particle is now described by a scalar and a vector. In total, a particle is described by a four component function. In electromagnetism, the electromagnetic fields $\vec{E}$ and $\vec{B}$ are vector fields. However, at the fundamental level, these two fields are represented by a scalar field $\varphi$ and a vector field $\vec{A}$, respectively. In this manner, at the fundamental level, a particle should be described by some similar fields, which are $\psi_{0}$ and $\vec{\psi}$ here. This makes the analogy between the field and particle representations symmetric. The electromagnetic wave is transverse, i.e., $\vec{E} \perp \vec{B} \perp \vec{k}$, while a particle wave is longitudinal.

In our formulation, this feature is very clear. Equation (6) states that the spin direction is along the direction of the field $\vec{\psi}$. As for photons, which are described by their polarization, a quantum particle should have some similar ansatz, where the spin is directly associated with the particle wave function. Thus, the wave function incorporates the spin states. In Dirac theory, the spin is deduced from the interaction of the electron with the photon magnetic field. In Schrödinger-Pauli theory, the spin of the electron is also deduced from their equation in the way the electron spin is coupled to the electromagnetic field. In ordinary quantum mechanics, the spin of the particle does not emerge from its wave function, but Equations (7) and (8) show that it does in our present formulation. 
In addition, if $\psi_{0}=-\vec{v} \cdot \vec{\psi}$, i.e., when $\vec{\psi}$ is projected along the direction of motion, then [6]

$$
i \hbar \frac{d \psi_{0}}{d t}=m_{0} c^{2} \psi_{0}, \quad i \hbar \frac{d \vec{\psi}}{d t}=m_{0} c^{2} \vec{\psi}
$$

The position and momentum commutation relation in quaternionic form now reads

$$
[\widetilde{X}, \widetilde{P}] \widetilde{\psi}=i \hbar \widetilde{\psi}
$$

where

$$
\widetilde{X}=(i c t, \vec{r}), \widetilde{P}=\left(i \frac{E}{c}, \vec{p}\right), \widetilde{\psi}=\left(\frac{i}{c} \psi_{0}, \vec{\psi}\right),
$$

where $\vec{\psi}$ and $\psi_{0}$ represent the quantum state of the particle in the quaternionic world.

The multiplication of two quaternions, $\widetilde{A}=\left(a_{0}, \vec{a}\right), \widetilde{B}=\left(b_{0}, \vec{b}\right)$, is given by

$$
\widetilde{A} \widetilde{B}=\left(a_{0} b_{0}-\vec{a} \cdot \vec{b}, a_{0} \vec{b}+\vec{a} b_{0}+\vec{a} \times \vec{b}\right) .
$$

Using Equation (3) and the fact that, in quantum mechanics $\vec{p}=-i \hbar \vec{\nabla}$ and $E=i \hbar \frac{\partial}{\partial t}$, and Equation (1) to get

$$
\begin{aligned}
& \vec{L} \cdot \vec{\psi}=\frac{3 \hbar}{c} \psi_{0}, \\
& \vec{L} \psi_{0}=3 \hbar c \vec{\psi},
\end{aligned}
$$

and

$$
\vec{L} \times \vec{\psi}=0 .
$$

Equation (5) states $\vec{L}$ transform the scalar wave function $\psi_{0}$ into the vector wave function $\vec{\psi}$. Notice, however, that, in quantum mechanics, $\vec{L} \times \vec{L}=i \hbar \vec{L}$. However, according to Equations (5) and (6), $\vec{L} \times \vec{L}=0$. We have recently developed the quaternionic quantum mechanics, but the physical meaning of the quaternion wave function remained unsorted [3,5,7].

Now, take the dot product of Equation (5) with $\vec{L}$ and use Equation (4) to get

$$
L^{2} \psi_{0}=9 \hbar^{2} \psi_{0} .
$$

Similarly, multiplying Equation (4) by $\vec{L}$ (from right) and using Equation (5) and the vector identity $\vec{A} \times(\vec{A} \times B)=\vec{A}(\vec{A} \cdot B)-A^{2} \vec{B}$, we obtain

$$
L^{2} \vec{\psi}=9 \hbar^{2} \vec{\psi}
$$

Hence, the quaternion components $\psi_{0}$ and $\vec{\psi}$ represent a state of a particle with a total orbital angular momentum of

$$
L=3 \hbar .
$$

This is a quite interesting result. It seems that this angular momentum arises from an internal degree of freedom. It may result from a rotation of some internal structure of the particle. In Dirac's theory, the spin of the electron doesn't emerge from the equation to be $1 / 2$, but is deduced to be $1 / 2$ from the way the electron interacts with the photon (electromagnetic field).

Now, differentiate Equation (5) with respect to time and use Equation (13) to obtain 


$$
\frac{d \vec{L}}{d t}=0,
$$

which implies that the orbital angular momentum is a constant of motion. It is thus a conserved quantity. Moreover, if we take the dot product of Equation (11) with $\vec{L}$ and use Equations (4), (5) and (10), then

$$
\frac{\partial \vec{L}}{\partial t} \cdot \vec{\psi}=0,
$$

and, if we use the fact that

$$
\frac{d \vec{L}}{d t}=\frac{\partial \vec{L}}{\partial t}+\vec{v} \cdot \vec{\nabla} \vec{L}
$$

then Equation (15) implies that

$$
\frac{d \vec{L}}{d t} \cdot \vec{\psi}=0 .
$$

Thus, either $\vec{L}$ is conserved or the external torque, $\vec{\tau}=\frac{d \vec{L}}{d t}$, is perpendicular to $\vec{\psi}(\vec{\tau} \cdot \vec{\psi}=0)$. Thus, the internal rotation of the particle is due to the self-interaction (internal constituents) of the particle.

Now, applying the condition, $\psi_{0}=-\vec{v} \cdot \vec{\psi}$, in Equations (4)-(6), and using Equation (9), yield

$$
\hat{L} \cdot \vec{v}=-c, \quad \hat{L}=\frac{\vec{L}}{L} .
$$

This shows that $\vec{L}$ is along the opposite direction of motion and that the particle (wave) moves with the speed of light. This is the group velocity representing the particle. This can be seen if we solve the system of equations, Equations (10)-(12). The wave equations representing the particle are

$$
\frac{1}{c^{2}} \frac{\partial^{2} \psi_{0}}{\partial t^{2}}-\nabla^{2} \psi_{0}+\frac{2 m}{\hbar} \frac{\partial \psi_{0}}{\partial t}-\left(\frac{m c}{\hbar}\right)^{2} \psi_{0}=0, \quad \frac{1}{c^{2}} \frac{\partial^{2} \vec{\psi}}{\partial t^{2}}-\nabla^{2} \vec{\psi}+\frac{2 m}{\hbar} \frac{\partial \vec{\psi}}{\partial t}-\left(\frac{m c}{\hbar}\right)^{2} \vec{\psi}=0,
$$

whose group velocity is $c$. Now let us choose

$$
\vec{\psi}=\frac{1}{c} \vec{\sigma} \psi_{0}
$$

where $\sigma$ are the Pauli matrices. In this case, $\vec{\psi}$ would represent a spin vector wave. Apply Equation (18) in Equations (5) and (6) to obtain

$$
\vec{L}=3 \hbar \vec{\sigma} .
$$

If substitute Equation (19) in the condition $\psi_{0}=-\vec{v} \cdot \vec{\psi}$, we will obtain

$$
\vec{\sigma} \cdot \frac{\vec{v}}{c}=-1
$$

This shows that the spin component is antiparallel to the direction of motion. This case agrees with that in Equation (18). Hence, the particle is left-handed! It seems that there is some internal degree of freedom (angular momentum) associated with quaternionic particles or, alternatively, that the quaternionic space has some twisting properties.

\section{Spin and Orbital Moment Interactions}

In this section, we discuss the application of the quaternionic form of the angular momentum on energy. Let us now consider the interaction energy $(U)$ of the magnetic dipoles in the presence of electromagnetic fields. This energy is due to spin $(\vec{S})$ and orbital angular momentum $(\vec{L})$, and the 
corresponding moments associated with them, are $\vec{\mu}_{s}$ and $\vec{\mu}_{\ell}$. The quaternionic form of the total angular momentum is defined as

$$
\tilde{J}=(0, \vec{L}-i \vec{S}) .
$$

The corresponding interaction energy can be written as [8]

$$
\tilde{U}=(i U, \vec{\tau})=-\tilde{\mu} \tilde{F},
$$

where

$$
\tilde{\mu}=\left(0, \vec{\mu}_{\ell}-i \vec{\mu}_{s}\right), \quad \tilde{F}=\left(0, \frac{\vec{E}}{c}+i \vec{B}\right) .
$$

When the dipole magnetic moment is placed in an external magnetic field, it experiences a torque $(\vec{\tau})$. The torque tends to align the dipole with (or opposite) the field. However, when the interaction energy is constant, the dipole moment precesses about the magnetic field. Substituting Equation (24) in Equation (23) and equating the real and imaginary parts of the resulting equations yield

$$
U=-\vec{\mu}_{\ell} \cdot \frac{\vec{E}}{c}-\vec{\mu}_{s} \cdot \vec{B}, \quad \vec{\mu}_{\ell} \cdot \vec{B}=\vec{\mu}_{s} \cdot \frac{\vec{E}}{c}, \quad \vec{\tau}=\vec{\mu}_{s} \times \vec{B}+\vec{\mu}_{\ell} \times \frac{\vec{E}}{c}, \quad \vec{\mu}_{\ell} \times \vec{B}=\vec{\mu}_{s} \times \frac{\vec{E}}{c} .
$$

Equation (25) encompasses all possible terms that may arise due to the presence of $\vec{\mu}_{s}$ and $\vec{\mu}_{\ell}$. The interaction of a magnetic moment with electric field has not been considered widely by physicists $[9,10]$, and hence this term is deemed to vanish. This term violates parity and time reversal invariance but respects rotational invariance [9]. Owing to duality between electric and magnetic fields, we trust that such terms should be present. It is shown by Aharonov-Casher that a phase shift occurs for a neutral particle with a nonzero magnetic dipole moment moving in an electric field [10]. This is given by

$$
\Delta \varphi_{S E}=\oint\left(\vec{\mu}_{S} \times \vec{E}\right) \cdot d \vec{r} .
$$

Using Equation (25), this is transformed into

$$
\Delta \varphi_{\ell B}=\oint\left(c \vec{\mu}_{\ell} \times \vec{B}\right) \cdot d \vec{r}
$$

This states that the effect of a spin magnetic moment in an electric field is equivalent to an orbital magnetic moment in a magnetic field. Aharonov-Bohm also showed that, in the absence of an electric field in the region, the phase shift of the particle wave function is given by

$$
\Delta \varphi=-q V \Delta t / \hbar,
$$

where $V$ is the electric potential and $\Delta t$ is the time spent in the electric potential [11]. In magnetic Aharonov, the phase shift is given by

$$
\Delta \varphi=q \phi_{B} / \hbar,
$$

where $\phi_{B}$ is the magnetic flux enclosed by the solenoid. The magnetic flux can be written as

$$
\phi_{B}=\int \vec{B} \cdot d \vec{A},
$$

but, if (from Kepler second's law)

$$
\frac{d \vec{A}}{d t}=\frac{\vec{L}}{2 m}=\text { const., } \quad \vec{A}=\frac{\vec{L}}{2 m} \Delta t,
$$

reflecting conservation of the orbital angular momentum in the closed loop, then applying 
Equations (30) and (31) in Equation (29) yields

$$
\Delta \varphi=\frac{q t}{2 \hbar m} \vec{L} \cdot \vec{B}=\frac{1}{\hbar} \vec{\mu}_{\ell} \cdot \vec{B} \Delta t .
$$

Comparing Equations (28) and (32) reveals that

$$
\frac{d \varphi}{d t}=\frac{q V}{\hbar} .
$$

Hence, in the absence of electric field, the charge behaves as a magnetic dipole interacting with a magnetic field. We thus may attribute the effect above to the spin and orbital angular momentum that the charge carries. It is of importance to remark that the rate of change phase in the Josephson junction, which is mediated by Cooper pairs, amounts to the same value in Equation (33) where $q=2 e$ is twice the electron charge [12].

\section{Conclusions}

Applying a quaternionic wave function, having scalar and vector components viz. $\widetilde{\psi}=\left(\frac{i}{c} \psi_{0}, \vec{\psi}\right)$, in the quaternionic commutator bracket for position and momentum operators, leads to an internal orbital angular momentum $L=3 \hbar$. In this model, this rotation could be attributed to the internal structure of the particle and hence to spin of the particle. Moreover, the vector part of the wave function, $\vec{\psi}$, points along the direction of $\vec{L}$. In the presence of an electromagnetic field, the electric and magnetic dipoles moments are found to be coupled to the field.

Author Contributions: All authors have contributed equally to this paper.

Funding: This research received no external funding.

Conflicts of Interest: The authors declare no conflicts of interest.

\section{References}

1. Bjorken, I.D.; Drell, S.D. Relativistic Quantum Mechanics; McGraw-Hill: New York, NY, USA, 1964.

2. Messiah, A. Quantum Mechanics; John Wiley: New York, NY, USA, 1968.

3. Arbab, A.I. The unifed quantum wave equations. Hadron. J. 2010, 33, 707.

4. Furey, C. Standard model physics from an algebra? arXiv 2016, arXiv:1611.09182v1.

5. Arbab, A.I. Derivation of Dirac, Klein-Gordon, Schödinger, diffusion and quantum heat transport equations from a universal quantum wave equation. Europhys. Lett. 2010, 92, 40001. [CrossRef]

6. Arbab, A.I. The analogy between matter and electromagnetic waves. Europhys. Lett. 2011, $94,50005$. [CrossRef]

7. Arbab, A.I. The quaternionic quantum mechanics. Appl. Phys. Res. 2011, 3, 160. [CrossRef]

8. Arbab, A.I. The complex Maxwell equations. Chin. Phys. B. 2013, 22, 030301. [CrossRef]

9. Anandan, J. Tests of Parity and Time-Reversal Noninvariance Using Neutron Interference. Phys. Rev. Lett. 1982, 48, 1660. [CrossRef]

10. Aharonov, Y.; Casher, A. Topological Quantum Effects for Neutral Particles. Phys. Rev. Lett. 1984, 53, 319. [CrossRef]

11. Aharonov, Y. Bohm, D. Significance of Electromagnetic Potentials in the Quantum Theory. Phys. Rev. 1959, 115, 485. [CrossRef]

12. Josephson, B.D. Possible new effects in superconductive tunneling. Phys. Lett. 1962, 251, 1.

(C) 2018 by the authors. Licensee MDPI, Basel, Switzerland. This article is an open access article distributed under the terms and conditions of the Creative Commons Attribution (CC BY) license (http:/ / creativecommons.org/licenses/by/4.0/). 